\title{
Two is better than one: combinatorial receptor targeting enhances hepatocellular carcinoma (HCC) therapeutic response
}

\author{
Celia Sequera, Flavio Maina \\ Aix Marseille Univ, CNRS, Developmental Biology Institute of Marseille (IBDM), Turing Center for Living Systems, Parc Scientifique de Luminy, \\ Marseille, France \\ Correspondence to: Flavio Maina. Developmental Biology Institute of Marseille (IBDM), Marseille, France. Email: flavio.maina@univ-amu.fr. \\ Comment on: Jin H, Shi Y, Lv Y, et al. EGFR activation limits the response of liver cancer to lenvatinib. Nature 2021;595:730-4.
}

Submitted Dec 07, 2021. Accepted for publication Dec 17, 2021.

doi: $10.21037 / \mathrm{hbsn}-21-517$

View this article at: https://dx.doi.org/10.21037/hbsn-21-517

Finding effective therapies for hepatocellular carcinoma (HCC) is an ongoing challenge for both researchers and clinicians. Enthusiasm for new treatment options emerging from in vitro, preclinical, and clinical studies is often tempered by limited patient benefits and associated side effects, tolerated in some cases due to the lack of alternatives. The complexity of identifying effective treatment options resides in several features of HCC, notably the extraordinary heterogeneity in molecular alterations among different patients (1-3), the lack of consistent alterations associated with oncogene addictions (4), and a so-called "non-oncogene addiction" by which tumorigenicity results from the action of non-mutated genes $(5,6)$. This complex configuration also prevents identification of unequivocal alterations used to design therapies, as for some other cancer types. Current HCC treatments are based on the Barcelona Clinic Liver Cancer (BCLC) guidelines according to the stage of lesions among other parameters. For HCC at early stages (30-40\% of HCC patients), treatments include lesion resection, liver transplantation, radiofrequency ablation (RFA) and microwave ablation (MWA), achieving a median overall survival (OS) of more than 60 months. For HCC at intermediate stages ( $20 \%$ of HCC patients), the advised treatment is generally transarterial chemoembolization (TACE), achieving an OS of 20 months. For HCC at advanced stages ( $40 \%$ of HCC patients), targeted therapies are the most frequent treatments, leading to an OS of 11 months with most targeted monotherapies. For HCC at terminal stages, only palliative care is provided $(\sim 10 \%$ of HCC patients), with less than 3 months OS $(7,8)$.
Over the last years, targeted HCC therapies introduced into the clinic have included multiple Receptor Tyrosine Kinase (RTK) inhibitors, such as sorafenib, cabozantinib, lenvatinib, and regorafenib. Limited responsiveness to these monotherapies is nevertheless a major problem, essentially due to primary or secondary resistance, the latter developing after treatment. Recently, a combined targeted therapy has gained particular attention and is currently used as a first line of HCC treatment, based on atezolizumab plus bevacizumab (an anti-PD-1 immunotherapy plus antiVEGFR), with a median OS of 19 months (9). Clinicians have even suggested that treatment options previously settled according to BCLC guidelines be revisited by applying this combinatorial treatment before ablation. Despite the high median survival rate of HCC patients following this immunotherapy, severe side effects have been reported in some cases. Therefore, new, potent treatment options are still needed for different HCC patient subgroups.

Jin et al. recently published in Nature the outcome of a synthetic lethality screen using a kinome-centred CRISPR-Cas9 library to identify genes driving lenvatinib resistance (10). The authors found that several guide RNAs (gRNAs) targeting the Epithelial Growth Factor Receptor $(E G F R)$ gene were depleted in the presence of lenvatinib, leading them to investigate the role of EGFR in lenvatinib resistance. Through a thorough set of complementary in vitro and in vivo studies, the authors showed that targeting EGFR confers responsiveness of HCC cells to lenvatinib. The combinatorial treatment effects consistently correlate with high levels of $E G F R$ expression $\left(E G F R^{\text {high }}\right)$, 


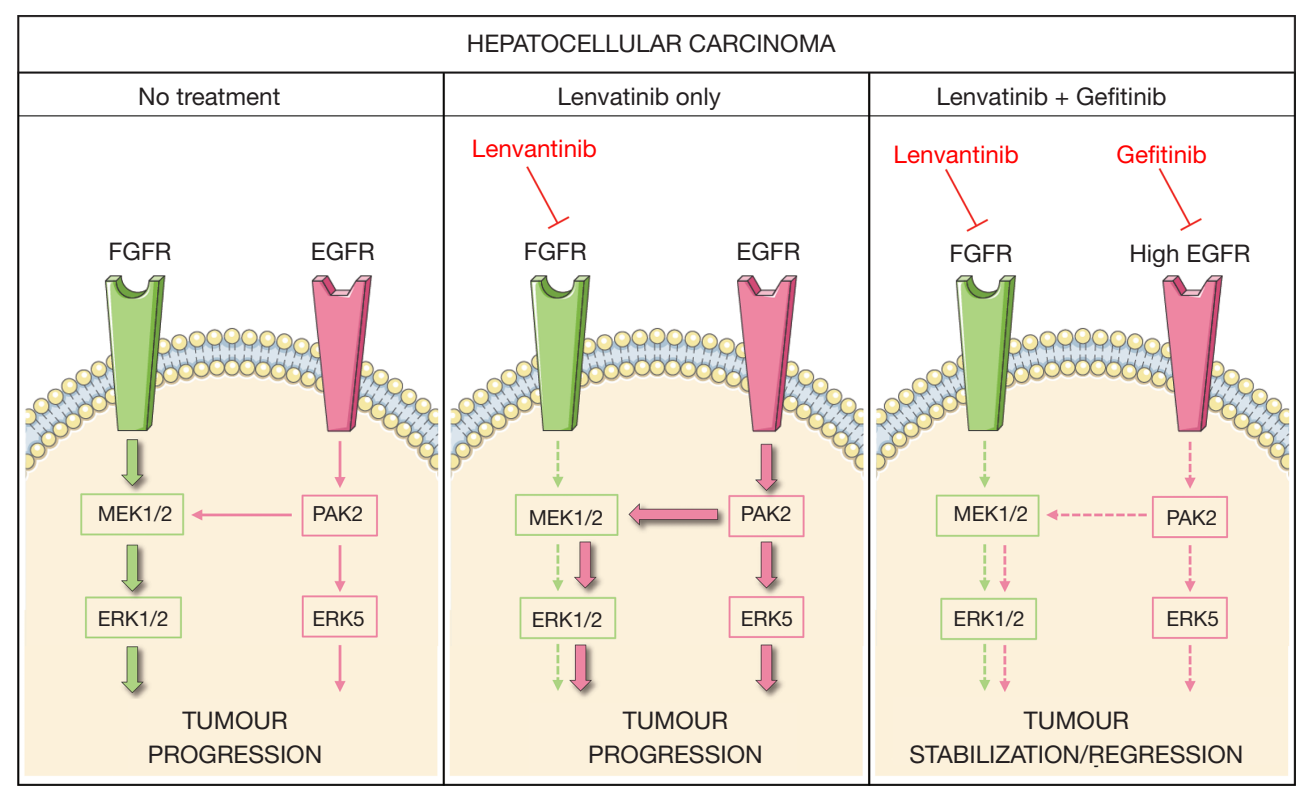

Figure 1 Schematic representation of the effects of lenvatinib alone and in combination with gefitinib on HCC cells, proposed by Jin et al. HCC, hepatocellular carcinoma.

as $E G F R^{\text {low }}$ HCC cell lines and patients do not respond. The authors revisited the results of their synthetic lethality screen to search for possible mechanisms underlying the effect of lenvatinib treatment while targeting EGFR. They found depletion of PAK2 and ERK5 gRNAs in cells treated with lenvatinib. The role of the EGFR/PAK2/ERK5 axis in lenvatinib resistance was investigated by a series of biological and biochemical studies (Figure 1), revealing its contribution among other mechanisms that likely remain to be uncovered.

The study by Jin $e t$ al. opens new possibilities to confer responsiveness of a HCC patient subgroup to lenvatinib through the simultaneous use of EGFR blocking agents like gefitinib or erlotinib. This combination may have the potential to limit intrinsic and acquired resistance over time, and to extend the range of patients for whom lenvatinib could be beneficial. Nevertheless, the work of Jin et al. also opens a number of intriguing questions that will be important to address in the future.

A first topic concerns the specificity in RTK targeting underlying this type of combinatorial strategy. The authors partially addressed this issue in relation to the spectrum of action of lenvatinib, which predominantly targets FGFR14, VEGFR1-3, PDGFR $\alpha$, KIT, and RET. Results showed that selective blockage of EGFR plus FGFRs recapitulates synthetic lethality, thus pointing to these RTKs as the main targets in the cellular contexts explored by Jin et al. (10). Additionally, they reported no significant effects of EGFR blockage plus sorafenib (predominantly targeting VEGFR2, VEGFR3, FLT3, PDGFR $\beta$, KIT, B-RAF, C-RAF), consistent with previous studies (11). In relation to other multi-tyrosine kinase inhibitors used in the clinic for HCC treatment, it would be particularly interesting to assess whether EGFR blockage elicits synergistic effects with cabozantinib (predominantly targeting MET, AXL, KIT, VEGFR2-3, RET, FLT3, ROS1, TYRO3, MER, TIE2) or with regorafenib (predominantly targeting VEGFR1-3, PDGFR $\beta$, KIT, FGFR, RET, CSFR, TIE2, B-RAF, C-RAF). Outcomes could be relevant to exploit the use of EGFR inhibitors in other combinatorial therapies for $E G F R^{\text {high }}$ HCC patients who may not respond to the lenvatinib + gefitinib combination. Furthermore, this issue of specificity could be relevant in relation to mechanisms of feedback activation following RTK inhibition, which can occur through: (I) signalling reprogramming via compensatory mechanisms [such as the EGFR/PAK2/ ERK5 pathway reported in this study (10)], (II) substitution by other RTKs to ensure signalling activation, an event named as "RTK switching" (12).

A second topic regards the proportion of tumours that respond to lenvatinib + gefitinib treatment and the degree of response. Jin et al. reported that response to 
this drug combination correlates with the $E G F R^{\text {high }}$ status, representing about $50 \%$ of HCC patients documented in this study. This may represent a valuable criterion to identify putative responding patients. Nevertheless, Jin et al. showed that not all EGFR ${ }^{\text {high }}$ tumours responded to the same extent. As HCC patients included in the study were previously treated with lenvatinib alone, the emergence of resistant clones cannot be ruled out. This configuration could be bypassed by the use of combined lenvatinib + gefitinib as a first line treatment. However, a secondary resistance may not be the only explanation for the partial response of some tumours. Indeed, Jin et al. showed that combined gefitinib plus lenvatinib treatment only partially reduced tumour growth in patient derived xenografts, as compared to the impaired tumour growth in HCC cell line xenografts. Ongoing clinical trial (NCT04642547) will clarify the robustness of the lenvatinib + gefitinib drug combination, possibly in relation to additional molecular features for future identification of responding patients. This would define supplementary criteria to narrow down the subgroup of HCC patients most likely to benefit from this combination, while redirecting $E G F R^{\text {high }}$ nonresponsive patients to more suitable treatments. Hence, it will be important to elucidate the specificity of action at the molecular and cellular levels, for example, in relation to the activation and expression levels of other RTKs. Outcomes may also uncover molecular mechanisms behind nonresponding $E G F R^{\text {high }}$ or $E G F R^{\text {low }}$ tumours. Additionally, it would be pertinent to assess whether pulses of lenvatinib + gefitinib treatment keep HCC under control over time, potentially limiting side effects and resistant clones emerging following a drastic depletion of responding cells. This configuration could allow transforming lesions into a chronic, controlled disease. Such configuration might be also relevant to the arising concept of non-genetic mechanisms of secondary resistance, which relies on the capacity of some drivers to confer plasticity to cancer cells to escape anticancer effects (13).

Another topic that deserves additional research concerns the effects of combined lenvatinib plus gefitinib therapy on the composition of immune cell types within the tumour microenvironment. In this regard, Jin et al. looked the presence of some immune cell types in relation to single and combined treatments. Among the changes reported, natural killer $(\mathrm{NK})$ and $\mathrm{CD}^{+} \mathrm{T}$ cells were increased in the combination treated tumours. Along this line, it has been reported that the EGFR inhibitor Icotinib elicited stronger effects in a subgroup of HCC patients with both high
EGFR and programmed death-ligand 1 (PD-L1) (14). A thorough characterisation of immune cell type composition associated with the combinatorial treatment would be highly informative to design pertinent immunotherapies, particularly for tumours in which regression is only partial.

Overall, the work of Jin et al. introduces a new combinatorial treatment option for HCC, the robustness of which as an effective therapy needs to be further documented. The new results also point to future possibilities by which the response to multi-kinase inhibitors currently used in the clinic may be enhanced by combining them with other agents, the potency and safety of which have been documented by their clinical use for other types of cancer. A major challenge that remains to be achieved is the stratification of HCC patients according to molecular signatures. This would maximize the choice of therapy options for HCC patient subsets, and reduce the risk that new treatment options will be discounted due to the inclusion of non-responsive patients in clinical trials.

\section{Acknowledgments}

We thank R. Kelly for extremely valuable feedback on this editorial.

Funding: CS was supported by a FRM fellowship. Maina lab is supported by research funding from Institut National du Cancer, ITMO Cancer Aviesan and INCa within the framework of the 2021-2030 Cancer Control Strategy on funds administered by Inserm, Cancerop le Provence Alpes C te d'Azur, Institute for Cancer and Immunology (AixMarseille University), GEFLUC-Les Entreprises contre le Cancer, and SATT Sud-Est.

\section{Footnote}

Provenance and Peer Review: This article was commissioned by the editorial office, Hepatobiliary Surgery and Nutrition. The article did not undergo external peer review.

Conflicts of Interest: Both authors have completed the ICMJE uniform disclosure form (available at https://hbsn. amegroups.com/article/view/10.21037/hbsn-21-517/coif). The authors have no conflicts of interest to declare.

Ethical Statement: The authors are accountable for all aspects of the work in ensuring that questions related to the accuracy or integrity of any part of the work are appropriately investigated and resolved. 
Open Access Statement: This is an Open Access article distributed in accordance with the Creative Commons Attribution-NonCommercial-NoDerivs 4.0 International License (CC BY-NC-ND 4.0), which permits the noncommercial replication and distribution of the article with the strict proviso that no changes or edits are made and the original work is properly cited (including links to both the formal publication through the relevant DOI and the license). See: https://creativecommons.org/licenses/by-nc-nd/4.0/.

\section{References}

1. Zucman-Rossi J, Villanueva A, Nault JC, et al. Genetic Landscape and Biomarkers of Hepatocellular Carcinoma. Gastroenterology 2015;149:1226-1239.e4.

2. Llovet JM, Zucman-Rossi J, Pikarsky E, et al. Hepatocellular carcinoma. Nat Rev Dis Primers 2016;2:16018.

3. Villanueva A. Hepatocellular Carcinoma. N Engl J Med 2019;380:1450-62.

4. Cancer Genome Atlas Research Network. Electronic address: wheeler@bcm.edu; Cancer Genome Atlas Research Network. Comprehensive and Integrative Genomic Characterization of Hepatocellular Carcinoma. Cell 2017;169:1327-1341.e23.

5. Nagel R, Semenova EA, Berns A. Drugging the addict: non-oncogene addiction as a target for cancer therapy. EMBO Rep 2016;17:1516-31.

6. Solimini NL, Luo J, Elledge SJ. Non-oncogene addiction and the stress phenotype of cancer cells. Cell 2007;130:986-8.

Cite this article as: Sequera C, Maina F. Two is better than one: combinatorial receptor targeting enhances hepatocellular carcinoma (HCC) therapeutic response. HepatoBiliary Surg Nutr 2022;11(1):139-142. doi: 10.21037/hbsn-21-517
7. European Association For The Study Of The Liver; European Organisation For Research And Treatment Of Cancer. EASL-EORTC clinical practice guidelines: management of hepatocellular carcinoma. J Hepatol 2012;56:908-43.

8. Arslanoglu A, Seyal AR, Sodagari F, et al. Current Guidelines for the Diagnosis and Management of Hepatocellular Carcinoma: A Comparative Review. AJR Am J Roentgenol 2016;207:W88-98.

9. Vogel A, Martinelli E; ESMO Guidelines Committee. Updated treatment recommendations for hepatocellular carcinoma (HCC) from the ESMO Clinical Practice Guidelines. Ann Oncol 2021;32:801-5.

10. Jin H, Shi Y, Lv Y, et al. EGFR activation limits the response of liver cancer to lenvatinib. Nature 2021;595:730-4.

11. Zhu AX, Rosmorduc O, Evans TR, et al. SEARCH: a phase III, randomized, double-blind, placebo-controlled trial of sorafenib plus erlotinib in patients with advanced hepatocellular carcinoma. J Clin Oncol 2015;33:559-66.

12. Maina F. Strategies to overcome drug resistance of receptor tyrosine kinaseaddicted cancer cells. Curr Med Chem 2014;21:1607-17.

13. Marine JC, Dawson SJ, Dawson MA. Non-genetic mechanisms of therapeutic resistance in cancer. Nat Rev Cancer 2020;20:743-56.

14. Sun J, Jiang W, Tian D, et al. Icotinib inhibits the proliferation of hepatocellular carcinoma cells in vitro and in vivo dependently on EGFR activation and PDL1 expression. Onco Targets Ther 2018;11:8227-37. 